suddenly released, it will be driven by the excess of pressure in the direction $\mathrm{O} \mathrm{H}$, and in that act the gas in $\mathrm{O}$ will be chilled and the gas in $H$ heated, which is contrary to the second law of thermodynamics, since in this process work is derived from matter all at a uniform temperature, or work is derived by cooling a portion of gas below the coldest of surrounding objects. In the same way the piston might have been connected to some external mechanism, and so part of the work be done externally (in a self-acting manner).

3. There can be little doubt that such work is done in natural processes (in the animal and vegetable world) since plants and organic tissues are distinguished for their porosity, and such tissues are permeated with the various gases of the atmosphere, carbonic acid, \&c. It may be observed that even without any porous diaphragm at all, or when two gases whose molecules possess different velocities are allowed to diffuse into each other, there is invariably a transference of heat, which is contrary to the second law of thermodynamics, which law assumes that heat cannot pass between two bodies originally at the same temperature, or heat cannot pass from a colder to a hotter body. Yet it is evident that as soon as the heat has begun to pass from one of the diffusing gases to the other, the one from which the heat commences to pass is already the colder.

4. Such a principle is evidently capable of an enormously wide application in nature. It is only necessary for example for the constituents of the universe to be diverse, to get any amount of work by diffusing them together, even if all originally at the same temperature. The principle of the tendency to the uniform diffusion of Matter, is capable of completely overthrowing the tendency to the uniform diffusion of Energy; for even if energy were uniformly diffused, the uniformity could be upset by the diffusion of matter (i.e. provided matter were not already all uniformly diffused or homogeneous) : and, as we have seen, the quantity of work to be derived by the diffusion of matter is limited only by the quantity of matter at disposal. ${ }^{1}$ In order that all capacily for work might cease in the universe, it would be necessary not only that there should be a uniform diffusion of energy, but also a uniform diffusion of matter. Heterogeneity confers a capacity for work, as well as inequality of temperature. Heterogeneity, as far as is known, is one of the distinguishing characteristics of the material universe. Any dissimilarity of molecular mass, which (by equality of temperature) is necessarily attended by dissimilarity of molecular velocity, confers a capacity for work. The dissimilarity of velocity is evidently the efficient cause in determining the work, and therefore in the exceptional case where dissimilarity of molecular structure is not attended by inequality of mass (and consequently not by inequality of velocity), work could not be derived. We may note, therefore, that inequality of molecular velocity, as well as inequality of molecular einergy, confers a capacity for work, and in order that all capacity for work should cease, not only must molecular energy', but also molecular velncity be uniformly distributed, or the molecules of matter which (by equality of temperature) possess unequal velocities, must be uniformly diffused.

5. We may observe that gravity which does not interfere with the uniform diffusion of energy, does interfere with the uniform diffusion of matter. Thus, for example, the energy (heat) of the atmosphere tends to be uniformly diffused throughout a vertical column of the atmosphere, in spite of the action of gravity. But the uniform diffusion of matter (i.e., the uniform mixture of the gases of the atmosphere through each other) is prevented by gravity. For by the well-known law of Dalton

${ }^{I}$ Since the first draft of this paper'was written, $I$ have been informed that the question of the quantity of work to be derived by diffusing gases has been treated of by Lord Rayleigh (Phil. Mag., April, 1875), but he does not apparently mention the bearing of the case on the'second law of thermo. dynamics. (which accords with the result of the kinetic theory of gases), each gas arranges itself as a layer upon the earth's surface, precisely as it would do if no other gas were present. Thus (as is known), owing to the fact that a greater quantity of nitrogen exists in the atmosphere than oxygen, the nitrogen consequently rises to a greater height than the oxygen, so that at considerable heights the nitrogen predominates. Thus the uniform diffusion of the constituents of the atmosphere through each other is prevented by gravity. It may, perhaps, be just as well "to note in connection with this point that those gases which are observed at the surface of nebula are not necessarily at the surface because of their greater lightness, but this is also determined by quantiiy; for as we have observed, each gas (according to the known conditions of equilibrium) arranges itself about a centre as if no other gas were present ; and therefore each gas. must penetrate to the centre of the nebula, and therefore could not reach as far as the surface unless its quantity were sufficient (though, no doubt, by a greater lightness a less quantity of gas will suffice for that purpose). There might possibly be a tendency to assume (unless the consequences of the above principle were rigidly kept in view) that the light gas observed (such as hydrogen) was floating on the surface of the nebula. We know that according to the conditions of gaseous equilibrium this is wrong, and that each gas (if freed from other disturbing causes) will have its basis at the centre of the nebula, where, therefore, the composition or mixture of gaseous matter is uniform, but nowhere else (excepting in the very improbable case where the quantities and densities of all the gaseous corstituents are the same). If gravity were to cease (and the gaseous constituents of the nebula were supposed confined or prevented from expanding), the constituents of the nebula would uniformly diffuse themselves throughout the entire mass, and this act of diffusion would be attended by a transference of heat, even if all the gaseous constituents were at the same temperature.

6. Thus we may observe that by merely modifying the action of gravity or by altering the position of a portion of gas relatively to gravity, work may be derived through diffusion. Thus if we suppose a portion of gas to be moved to different positions in a nebula, the constitution of the portion of gas or the mixture of its constituents is changed according to its position, and in these changes work is derived, or available. Only when the portion of gas is situated at the centre of the nebula are its constituents uniformly diffused through each other; less and less so towards the outside.

7. It would thus appear to follow that, as far as present knowledge goes, a uniform diffusion of matter as well as a uniform diffusion of energy would be at least required, in order that all capacity for work and physical change should cease in the universe. At the same time does it not rather behove us to look to a time when, through increase of knowledge, a means for recurrence may possibly be discovered, whereby physical change is continued, rather than to look to the purposeless end of a chaos of uniform temperature and uniform distribution of matter? Humboldt says relatively to this point (Preface to "Cosmos"): "I would therefore venture to hope that an attempt to delineate nature in all its vivid animation and exalted grandeur, and to trace the stible amid the vacillating ever-recurring alternation of physical metamorphoses, will not be wholly disregarded at a future age."

S. TOLVER PRESTON

\section{MUSIC A SCIENCE OF NUMBERS}

$T H E$ subject which I submit for your consideration this afternoon is the influence of numbers in music, as in the various combinations of consonances and dissonances i. Read before the Musical Association of London, November 5, 1877 , by 
which we hear every day, and to show how these are explained by the fundamental laws of the science.

Although music has appeared to many persons a difficult subject, it is really one of the most easily intelligible and one of the most firmly grounded of sciences. It is purely a science of numbers.

The consonances which charm the ear, such as the octave, twelfth, fifth, fourth, and the major and minor thirds, have two concurrent sets of vibrations; the one set produced by the lower string or pipe, and the other by the upper. Although they vibrate at different rates, yet there are periodical coincidences of vibration between them, and these coincidences sound with much more power upon the ear than the vibrations which are noncoincident, or sound apart. It has been calculated that two hammers striking simultaneously upon an anvil have, through the greater displacement of air, fourfold loudness, instead of merely double. The same law applies to musical sounds. Coincidence of vibration is more briefly expressed by its synonym, "consonance;" and all noncoincident vibrations are included in "dissonances," meaning only that they sound apart. In a musical sense, dissonance is the medium between concord and discord, running from one into the other; for, in the most pleasing intervals, there are some non-coincident vibrations, and when these become very numerous, they overpower all concord. This will be shown in the sequel.

Suppose we take one long pianoforte string or an organpipe. The lowest sound it can produce will be that of its whole length, and this may be made the foundation of an entire scale of consonant notes, for every aliquot part of the length, being such as will measure without any remainder, will be also a multiple of the vibrations of No. I. Thus No. 2, the octave, is half the length and vibrates twice as fast as the whole string. No. 3, the so-called twelfth, or octave and fifth, is a third of the length of No. I, and it vibrates thrice as fast. Then, if we sound No. 3 with No. 2 instead of No. I, we throw off the lower octave and have the fifth only, or 3 to 2 . It is essential for consonance that the intervals should be aliquot parts of No. I, for if otherwise, we should only create discord. The musical law is expressed very simply, that the number of vibrations is in inverse ratio to the length of a string.

The scale of all consonances is called the harmonic scale, copies of which are before you. It is exemplified by string or pipe. Let us consider, first, the Eolian harp, on which the winds alone produce the consecutive sounds. The strings are tuned in unison, except the two outmost, one on each side, and those are covered with wire, and tuned an octave lower. When the wind blows quickly enough to sound the bass strings, which we will suppose to have tuned to $\mathrm{C}$ on the bass elef, with 128 vibrations in a second of time, it is the whole string which sounds first, and the rapidity of the wind must be doubled before the harp will sound any change of note, and that note will be the octave above the first. It has already been said that the octave is produced by half the length of a string, and that it vibrates twice as fast as the whole-but mark the coincidence between the music and consecutive numbers; $I$ and 2 have no note between them, although the sound jumps from the whole length to that of the half! When the bass strings sound the half length they have divided themselves into equal halves by a node, and that node creates tension in opposite directions, the one ventral segment pulling, as it were, against the other. These self-forming nodes may be easily seen by daylight, and at night by throwing a light upon the string. They were shown at our first conversazione in these rooms by Mr. Spiller, and at the Edinburgh meeting of the British Association by Mr. Ladd. The gust of wind which sounds the octave, or half length of the bass strings of the Æolian harp, sounds at the same time the whole length of the gut strings, because they are tuned to that pitch. Then, as the wind rises, subdivision goes on in both with every multiple of 128 vibrations for the bass, and of 256 vibrations for the tenor strings.

The reason for tuning the Æolian harp to a low pitch is, that the strings may be more easily acted upon by the wind. We read, poetically, of hanging one in a tree, but it requires a much stronger draught than it will get there, except during a hurricane, when no one will care to go to listen. Our late lamented Vice-President, Sir Charles Wheatstone, F.R.S., fixed a single violin string under a very draughty door, as an Æolian harp, and he calculated the increase of draught caused by lighting a fire in the room, and by the opening of an outer door, by the rising pitch of the note. The varieties produced by this string have been described as "simultaneous sounds," but they were purely consecutive. Anyone may satisfy himself that it could only be so, by repeating the experiment with a good violin string. The change of note is simultaneous with the change of nodes in the string. Mere undulations, or irregularities of vibration, will not change the note, but injure the quality of the tone. All the curves that a string may describe in vibration have been calculated by mathematicians, but only when nodes are formed are they of any importance in music.

Often have I experimented upon harmonics or natural sounds, in former years, and have watched the changes of node, and have heard the simultaneous change of note. The experiments may be tried by any one who has access to a harpsichord, or a very old grand pianoforte. The tension is too great in modern instruments to allow free play to the string. Raise the damper and strike one of the longest uncovered strings with a hard pianoforte hammer near the bridge. The changes follow in numerical order, $1,2,3,4,5$, as in the paper before you, and the sounds ascend by octave, fifth, fourth, major and minor third, harmonic seventh, to the third octave, and then to the major and minor tones. It is difficult to attain the highest of these numbers, but the harmonic seventh, No. 7, is readily distinguished by its unusual sound.

In the Eolian harp the rising pitch of the sounds is caused by the increasing rapidity of the wind; but it is not so on a pianoforte. It is there due to gradual contractions of the string till it ceases to vibrate, and sinks to rest. The vibrations of a long string are widely discursive, but they become gradually more and more contracted as the nodes of the string diminish in length. The point to be remarked is that the sounds jump over intermediate discords - all are consonances---all aliquot parts : all the sounds are multiples of No. I. It matters not whether it be wind, string, or pipe; in each of them nature teaches us the scale which is to resolve all musical doubts, all disputed chords. She indicates all the basses for musical intervals, the more remote ones adapted only for melody, and the nearest for consonant harmony.

To prove the case further we may take an illustration from a pipe. It must not be from those which have lateral openings, or keys, because they shorten the column of air artificially, but from such instruments as the coach horn, or hunting horn, the so-called French horn, or the trumpet without valves.

The fundamental tone, No. I, or lowest sound it can produce, is derived from the whole column of air within the tube. To produce No. 2 the rapidity of the breathing must be doubled, and then the column of air within the horn divides itself into two equal halves, and the sound is an octave above ; so that, if the first note be tenor $\mathrm{C}$ with 256 vibrations in a second of time, this treble $C$ requires to be blown at the rate of 256 vibrations to produce it. Here, again, we arrive at the identification of sounds with numbers ; for, just as there is no intermediate number between $I$ and 2 , so is there no intermediate sound between $I$ and 2 , its double in vibrations, produced by half its length, upon the horn. The 
numbers run both ways. They are fractions as to length of tube, and multiples as to vibrations. Again, just as there is an intermediate number between 2 and 4 (the second octave), so is there one intermediate sound, and one only; it is No. 3, which is produced by a third of the length of the tube, and is the fifth above No. 2. The fifth and fourth divide the vibrations of the octave equally between them, so that the fifth is three times No. I, and the fourth immediately above it is four times;-this, notwithstanding the diminution of the musical interval. The names which we have adopted for musical intervals are usually calcúlated from the keynote, as from $\mathrm{C}$ to $\mathrm{E}$ a third, from $\mathrm{C}$ to $\mathrm{F}$ a fourth, and from $C$ to $\mathrm{G}$ a fifth, but these names are not real quantities, and are rather confusing than an assistance. The octave is not an eighth, but half, and the double octave is not a fifteenth, but a quarter of the length of No. I, and vibrates four times as fast. Octaves are powers of 2 , thus $2,4,8,16$, and 32 are successive octaves. But the octave 4 to 8 has only four sounds, and these are our major and minor third, and two others, divided by the harmonic seventh, which we do not use. From 8 to 16 are eight sounds, of which we use three, the major and minor tones, and the so-called diatonic semitone, as from B to $C$. It is really the smallest of the eight tones, and not a semitone. The next octave is from 16 to 32 , and that is all of semitones, while 32 to 64 is all of quarter-tones. After that, the octave is divided into eighths, sixteenths, and thirtysecond parts of tones, among which it is only useful to note (and that only among musicians and mathematicians, that the so-called "comma," having the ratio of 80 to $8 \mathrm{I}$, is the eighth of a tone above the third of any key-as it is above $\mathrm{E}$ in the key of $\mathrm{C}$. We have lately had mathematicians among us who are not $\mu$ ovoıкoi, and who have, therefore, proposed to divide an octave into "twelve equal semitones." This is pure geometry, and not music. In music there cannot be even two equal semitones within an octave. If our friends will only change their theme from twelve equal semitones into twelve equally tempered semitones, and give us their experience of the proposed sounds when heard with the bass (which seems not to have yet been taken into account), we shall gladly avail ourselves of their research, on the grounds of modern expediency. In the meantime we must be content to leave the tempering of a scale in the hands of experienced practical men, who, judging only by their ears, as they always will, have hitherto satisfied our immediate requirements.

The interval of a fifth is 2 to 3 in ascending and 3 to 2 in descending, but, as the figures are usually placed over the upper note in scales, the 3 is written above the 2 as in the scale in your hands (the third of them), where it appears over $\mathrm{G}$, referring to $\mathrm{C}$ as 2 .

And now for the practical use of these figures, for although the harmonic scale may be referred to, they are most easily remembered. All young pupils are taught the difference between an octave, a fifth, a fourth, and a third, upon the pianoforte, and it is only to associate the numbers with those intervals, to find out the best bass, and every admissible bass. All octaves are in the ratio of 2 to $\mathrm{I}$, whether it be 4 to 2,8 to 4 , or 16 to 8 . All fifths are in the ratio of 3 to 2 , all fourths in that of 4 to 3 , all major thirds 5 to 4 , and minor thirds 6 to 5 .

For instance, in the key of $\mathrm{C}, \mathrm{C}$ to the $\mathrm{F}$ above it is a fourth, and $\mathrm{F}$ is No. 4, therefore, the F, two octaves below, is the consonant bass; whereas, if we strike $G$ with the $\mathrm{C}$ above, $\mathrm{C}$ becomes the natural bass to that interval. The most consonant basses are always found in the lowest numbers, because the proportion of consonant vibrations is there greatest. Thus, from $\mathrm{D}$ to $\mathrm{G}$ is also a fourth, in the key of $C$, but the numbers are 9 to 12 , with a remote bass in $C$, and there will be $2 \mathrm{I}$ vibrations, of which only two will coincide in every cycle-I of the 8, with I of the 9. Then, the proportion of noncoincidence will be so great as to make the sound unpleasing to the ear. But as 9 to $\mathrm{I} 2$ is in the ratio of 3 to 4 , we have the best bass in these lowest numbers, and take $G$. By the various basses to intervals we modulate into other keys.

At the International Exhibition, held at South Kensington in $1862, \mathrm{Mr}$. Saxe, the eminent inventor of Saxe horns, exhibited an immense horn with an exceedingly long coil of tube, and perhaps standing six feet in height. When asked by the jury the object of this excesssive size and length, he answered, "C'est pour jouer dans le cinquième étage ".." It is for playing in the fifth octave," and he produced with facility any of the sixteen tones and semitones of that octave from it. Half the length of any open conical tube is expended upon its second note, the octave. No human power could have blown the low notes of that horn. Supposing it to have been tuned to the lowest $\mathrm{C}$ upon the pianoforte, with thirty-three vibrations in a second, as the usual French pitch, it would have had 66, I32, 264, and 528 for its first, second, third, and fourth octaves, while its fifth octave would commence on treble $\mathrm{C}$, with 528 , and extend to $\mathrm{C}$ above the lines with 1056 vibrations in a second of time. It would thus be within the power of the lungs. $\mathrm{He}$ utilized only from the 16 th to the 32 nd part of his enormous tube, but it gave him the command of the semitones.

This great incumbrance of length is not necessary in a cylindrical stopped tube. It will take up its own octave according to the ratio of its length to its diameter. We have here an example in a resonating tube invented by Charles Wheatstone just fifty years ago. The lecture for which he invented it was afterwards reported in the twenty-fifth volume of the Quarterly Fournal of Science, Literature, and Art, January to March, I828. Both he and I knew Eulenstein, an accomplished musician, whose admirable skill in playing upon the Jew's harp was the inducing cause of that particular lecture. Eulenstein had a peculiar facility for contracting and expanding the cavity of his mouth, through the pliability of his very thin cheeks and by the management of his tongue, so that he could fit them for any harmonic note within a certain compass. Wheatstone then gave the law, that a perfect harmonic scale might be drawn from a single tuning-fork, or from the vibrating tongue of a Jew's harp, by resonators adapted, or adapting themselves, to multiples of the original number of vibrations. "I took," said Sir Charles, "a tube, closed at one end by a movable piston, and placed before its end the branch [or prong] of a vibrating tuningfork of the ordinary pitch-C. The length of the column of air [within the tube] was six inches. On diminishing the length of the column of air to three inches [by moving up the piston], the sound of the tuning-fork was no longer reciprocated [in unison], but its octave was produced." "It is therefore evident from experiments," says he " that a column of air may vibrate by reciprocation, not only with another body whose vibrations are isochronous [or in unison] with its own, but also when the number of its own vibrations is any multiple of the sounding body." Again, be says: "No other sounds can be produced by reciprocation from a column of air, but those which are perfectly identical with the multiplications of the original vibrations of the tuning-fork or the tongue of the Jew's harp." I produced the original tube in this room about two years ago, to check a recent theory-that resonators strengthened the ear, and answered only in unison, and Sir Charles ordered this one for me, made by Mr. Groves, under his own superintendence. The improvement in this is, that the piston now works in a groove and is not liable to stick. Two octaves are produced from the tongue of one Jew's harp as rapidly as the piston can be moved up and down. There is 
no slurring between one sound and another, but clear jumps from one multiple to another, and every one of them may be arrested and heard by itself by checking the piston. But, although I am glad to produce this tube before those who were not present on the last occasion, and to do honour to the memory of our eminent vicepresident, who declined to refer in any way to himself, I have another motive also. This is a principle which has never been utilised. We have had pipes stopped at the top, like the usual pitch-pipe, but they have been found too slow in action to be suitable for any other purpose. This is rapidity itself, and might surely be utilised for some such purpose as pedal-pipes for an organ. The piston can be balanced outside to the greatest nicety, and one such pipe will take the scale of $C$, and another that of F. All that is required is to blow across the top in the manner of the Pandean pipes, or, as it appears, better still, to set free a fan or cogged wheel at the mouth tuned to each of the two fundamental notes. The wheel might be set free by the action of the foot upon the pedal. It is now well known that the length of a 32 or a 16 foot pipe may be greatly reduced by breadth of scale. We Europeans have made little, if any, use of resonators, and yet they have been long in use in Java. The drawing on the wall is of an instrument brought from Java by Sir Stamford Raffles more than half a century ago. There is one of the same kind in the British Museum. But this is perhaps of greater interest, as it may have suggested to Wheatstone the pronciple of the resonating tube. The natives of Java cast metal plates which they suspend in a row upon strings, and strike them with drum-sticks, which are fitted into circular heads. As all cast metal is more or less false in tone, owing to inequalities and lack of homogeneity, they place some of the largest bamboos, cut to short lengths, and placed upright, under the metal to make the true sounds of these resonators to overcome the false harmonics of the metal plates.

Resonators were used in the theatres of ancient Greece -we here find them used in Java; but these powerful auxiliaries to tone still await their development in modern Europe.

And now, in conclusion, permit me to draw your attento a harmonium with two keyboards, the upper one having four octaves of our scale tuned without tempering, and the lower with the five octaves of the harmonic scale, and the sixteen noces in the fifth octave. Much has been said of the harmonic scale, and this is perhaps the only instrument on which the harmonics can be fully heard and sustained for experimental use.

\section{ROBERT SWINHOE, F.R.S.}

W ITHIN the last thirty years or so their respective vocations happen to have called two able lovers of natural history in the direction of the Celestial Empire -Mr. Robert Swinhoe, from England, and the Père Armand David, a Frenchman. The simultaneous investigations of these two biologists have added immensely to our knowledge of a country whose fauna not long ago was thought to be in no way interesting, because the huge population had succeeded in extirpating all the indigenous species. How far from the truth such an assumption is, has been demonstrated by the researches of the two naturalists above mentioned, the lamented death of the former of whom, at the early age of forty-one years, we recorded last week.

Mr. Swinhoe was born at Calcutta on September $t_{\text {, }}$ 1836 , and was educated at King's College, London, whence he matriculated at the University of London, in 1853. The next year he went, as supernumerary interpreter, to Hong Kong, being transferred to Amoy in I855, and to Shanghai in 1858 . In the same year he was attached to the Earl of Elgin's special mission to China, and afterwards to H.M.S. Inflexible as interpreter in a circumnavigating expedition round Formosa, in search of certain Europeans said to have been held in captivity at the sulphur mines on the island.

In $1860 \mathrm{Mr}$. Swinhoe attended Gen. Napier, and afterwards Sir Hope Grant, the Commander-in-Chief, as interpreter, and received a medal for war service. At the end of the same year he was appointed Vice-Consul at Taiwan, Formosa, and in 1865 to the full Consulship. In 1866 he was Consul, temporarily, at Amoy, and in 1868 went to explore the Island of Hainan. From May, I87I, to February, 1873, he was acting Consul at Ningpo, and at Chefoo until October of the latter year, when he had to retire from the service, on account of increasing paraplegia, from which he died on October 28 last.

Mr. Swinhoe was a Fellow of the Asiatic Societies of China and of Bengal, as well as of many other societies, having been elected into the Royal Society in 1876 .

By far the majority of Mr. Swinhoe's scientific communications-fifty-two in number-mostly on the mammalia and birds of China, are to be found in the Proceeding-s of the Zoological Society of London-between I86I and 1874. Other papers appeared in the Ibis and the Annals and Magazine of Natural History within the same period. Among the most important of these are the "Catalogues" of the mammals and birds of China and its islands, in which are to be found descriptions of many new species of both classes, among which are St. John's Macaque (Macains sancti-johrinnis), the Water Deer of Shanghai (Hydropotes incmuris), the Mantchurian Deer (Cervus mantchuricus), the Orange-bellied Helictis (Helictis subaurantiaca), the Superb Flying Squirrel (Pteromys grandis), Boyce's Stork Ciconia boyciana), together with a great number of other birds, for a complete account of which we cannot do better than refer our readers to a work upon the birds of China, by M. l'Abote David and M. E. Oustalet, published at Paris a week ago.

Michie's Deer (Lophotrayus michianus) is the name given by Mr. Swinhoe to a small deer from Ningpo, with antlers more diminutive than many other species. This, or a very closely-allied species, was previously sent to Paris by Père David, and described by M. A. MilneEdwards under the name Elaphodiss cephalophis.

Mr. Swinhoe, besides the collections which he made, was indefatigable and particularly successful in his endeavours to send living animals from China to this country, and there are many species, including Cervuts swinhoii, Hydropotes incruris, and Ciconia boyciana. which were first procured by him.

It will be some time, we fear, before so enterprising a naturalist as Mr. Swinhoe takes up his residence in China, and employs every available opportunity for the prosecution of his favourite line of research.

\section{DOUGLAS A. SPALDING}

UR readers 'must be familiar with this name as that of an occasional contributor to NATURE of thoughtful and acute articles in the department of mental science; they will be sorry to hear-but those who knew him will not be surprised-that Mr. Spalding died on October 31, at Dunkirk, just as he was preparing to go to the Mediterranean coast to spend the winter. Not much is known of Mr. Spalding's early life, but we are told by one who ought to know that his parents, belonging to Aberdeenshire, were in very humble circumstances, and that he was born in London about the year 1840 . He himself spent his early years in Aberdeen as a working slater, doing his best to educate himself, By the kindness of Prof. Bain Mr. Spalding was allowed to attend the classes of Literature and Philosophy in Aberdeen University free of charge, in the year 1862. After that he got some teaching about London, and worked very hard to support himself, and even managed to keep his 